Ann. Sci. forest., 1981, 38 (1), 107-126

\title{
Technique de microcuissons papetières : perfectionnement et précision des rendements en pâte
}

\author{
G. JANIN \\ I.N.R.A., \\ Station de Recherches sur la Qualité des Bois \\ Centre de Recherches de Nancy \\ Champenoux, 54280 Seichamps
}

\section{Résumé}

Les perfectionnements apportés à la méthode de microcuisson correspondent à la construction d'un nouveau bain d'huile à régulation de température programmable avec un système d'agitation et d'entraînement de 48 microlessiveurs disposés sur un même support en rotation dans l'huile et à l'amélioration du mode opératoire.

Un micro-indice de permanganate $\mathrm{KMnO}, \mathrm{N} / 10$ a été établi pour définir la qualité des pâtes obtenues en microlessiveurs. La présence tout à fait exceptionnelle d'incuits renforce la validité des comparaisons entre échantillons. La précision relative du rendement en pâte est de $\pm 0,1 \%$ à la suite des pesées, tous les autres paramètres étant constants.

Au cours d'expériences conduites en routine, la reproductibilité du rendement en pâte et la possibilité de sélectionner des familles ou de choisir des provenances possédant les meilleurs qualités papetières ont été éprouvées. Les influences des facteurs usuels de variation des cuissons papetières ont été testées, ainsi que les effets de la position et de la forme du prélèvement des échantillons dans un Populus tremula.

Les correspondances entre les résultats de cuissons d'échantillons de faible poids $(0,5$ à $1 \mathrm{~g})$ et de taille plus importante $(200 \mathrm{~g})$ sont satisfaisantes $(\mathrm{r}=0,832)$.

Le comportement identique de parallélépipèdes de bois découpés au voisinage du prélèvement de carottes de sondage est au bénéfice de ces dernières qui permettent de pratiquer, à l'aide d'essais non destructifs pour les arbres, les déterminations des caractéristiques papetières.

\section{1. - Introduction}

La méthode de microcuisson est une technique de laboratoire qui a été mise en œuvre pour reproduire, à une échelle très réduite, les procédés industriels de cuisson des bois ou de tous autres végétaux pour la fabrication de la pâte à papier (E.F. THOdE et al., 1961; J.L. KeAYs et al., 1969 ; G. JANin, 1972 a ; J.F. Manville et K. Hunt, 1977).

La cuisson papetière est un traitement du bois ou de végétaux par des solutions délignifiantes alcalines ou acides en vue de séparer les fibres en dissolvant partiel- 
lement les lignines et en conservant en «l'état» le mieux possible la cellulose et les hémicelluloses des membranes cellulaires.

Le rendement de la cuisson qui, pour un traitement donné, est le rapport du poids des fibres isolées anhydres au poids de bois anhydre, constitue un indice de qualité du bois pour les essences papetières car, pour une même biomasse récoltée, un rendement plus élevé signifie une production accrue de pâte à papier.

La détermination précise du rendement en pâte de nombreux échantillons de bois de petite taille, souvent inférieurs au gramme, prélevés de façon non destructive dans les arbres vivants à l'aide de la tarière de Pressler de $5 \mathrm{~mm}$ de diamètre, est nécessaire si l'on veut tenir compte de la variabilité individuelle des arbres; clle nécessite une fiabilité et une bonne reproductibilité des conditions opératoires afin de s'affranchir de toutes les sources de variations autres que celles concernant la nature et lorigine des échantillons examinés lors des comparaisons entre individus et de l'estimation de l'héritabilité de ce caractère (D.W. EinsPaHr et al., 1962, 1969 ; R. BLAir et al., 1975).

La technique de microcuisson n'est pas réservée uniquement à la mesure précise du rendement en pâte. En effet, elle peut être utile à la détermination des indices usuels de la qualité des pâtes : dosage des liqueurs noires de fin de cuisson pour évaluer la consommation des réactifs, l'indice de Kappa ou de permanganate en relation avec la «dureté » de la pâte pour l'aptitude au blanchiment, la teneur en lignines résiduelles, en pentosanes et en cellulose de la pâte, ainsi que la longueur des fibres et la courbe biométrique de répartition pour chaque échantillon.

Dans l'industrie, il existe des procédés de cuisson en continu dans des lessiveurs de très grande capacité, ainsi que des procédés en discontinu avec remplissage de bois et vidange totale de la pâte à chaque opération. Notre méthode se rattache à ce dernier type de cuisson.

\section{2. - Matériel et méthode}

Bien que tous les types de cuisson puissent s'employer pour nos microcuissons, nous pratiquons presque exclusivement des cuissons alcalines dites «au sulfate» du type "Kraft», c'est-à-dire avec un mélange de soude et de monosulfuture de sodium avec les caractéristiques suivantes:

- l'alcali actif $(\mathrm{NaOH}+\mathrm{Na}, \mathrm{S})$ exprimé en p. $100 \mathrm{du}$ poids de bois anhydre,

- la sulfidité, qui est le rapport $\mathrm{Na}_{2} \mathrm{~S} /\left(\mathrm{Na}_{2} \mathrm{~S}+\mathrm{NaOH}\right)$,

- le rapport de dilution : volume du liquide (en $\mathrm{ml}$ )/poids de bois en grammes, qui règle la concentration des produits actifs,

- le diagramme température - temps variable.

Les aménagements apportés à la méthode de 1972 (G. JANIN, op. cit.) concernent, d'une part, le matériel pour permettre de traiter un plus grand nombre d'échantillons dans des conditions comparables au cours d'une même cuisson et entre cuissons successives, et d'autre part, le mode opératoire pour obtenir une plus grande précision, en particulier de façon à minimiser l'effet «opérateur». 


\section{1. - Matériel}

\subsection{1. - Bain d'huile (figure 1)}

Nous disposons d'un bain d'huile d'un volume de 35 litres avec une puissance installée de $4,5 \mathrm{~kW}$ pour permettre des montées en température en fonction du temps commandées par un régulateur à came. Une hélice assure le brassage de l'huile pour homogénéiser la température. Il peut recevoir deux types de lessiveurs, ce qui nous assure des conditions opératoires identiques nécessaires à la comparaison de traitements, soit :

- 48 microlessiveurs disposés sur un support rotatif tournant à une vitesse de 6 tours par minute pour assurer l'agitation des réactifs, et aussi une homogénéisation supplémentaire des températures entre lessiveurs,

- 4 macrolessiveurs placés sur un support différent, mais agités de la même façon.

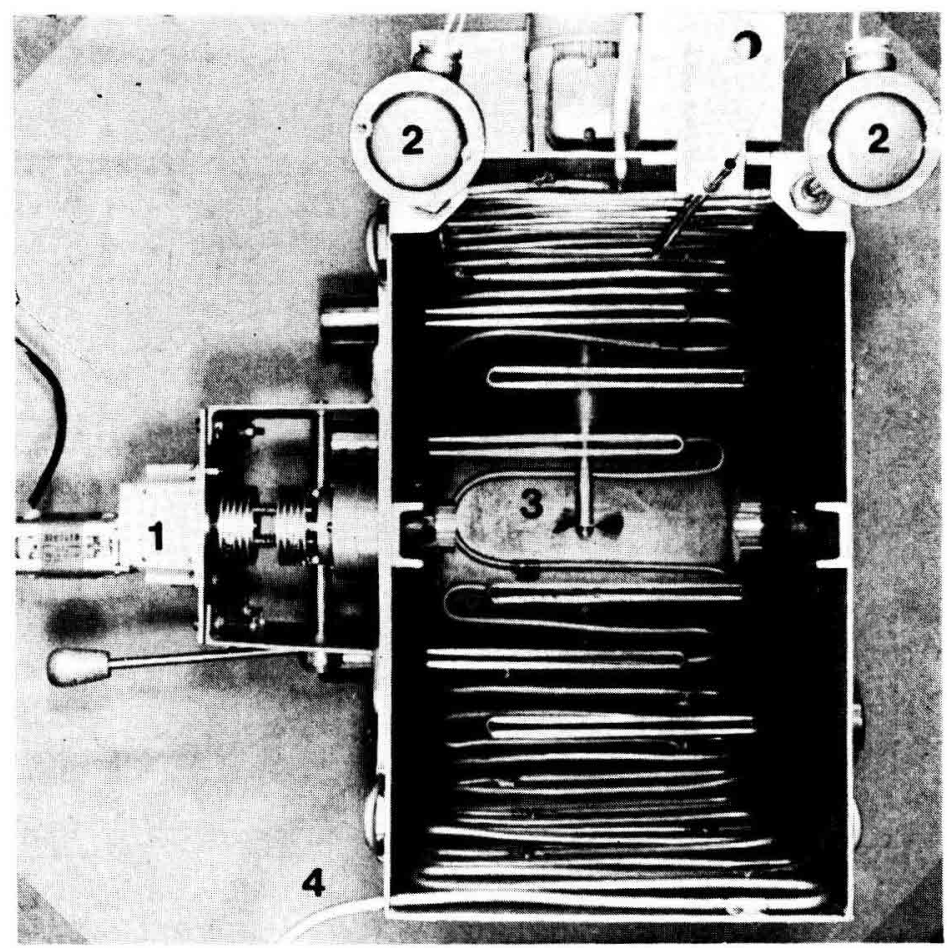

FIG. 1

(Cliché Durand)

Bain d'huile ( $v u$ de dessus, couvercle retiré)

1 : moteur d'entraînement du support des microlessiveurs,

2 : sondes de température et de régulation,

3 : hélice,

4 : circuit de refroidissement de l'huile.

Oil bath (upper-view, cover removed)

1 : motor drive for rotating wheels,

2 : temperature and regulator gauges,

3 : propeller,

4 : oil cooler copper tubing. 


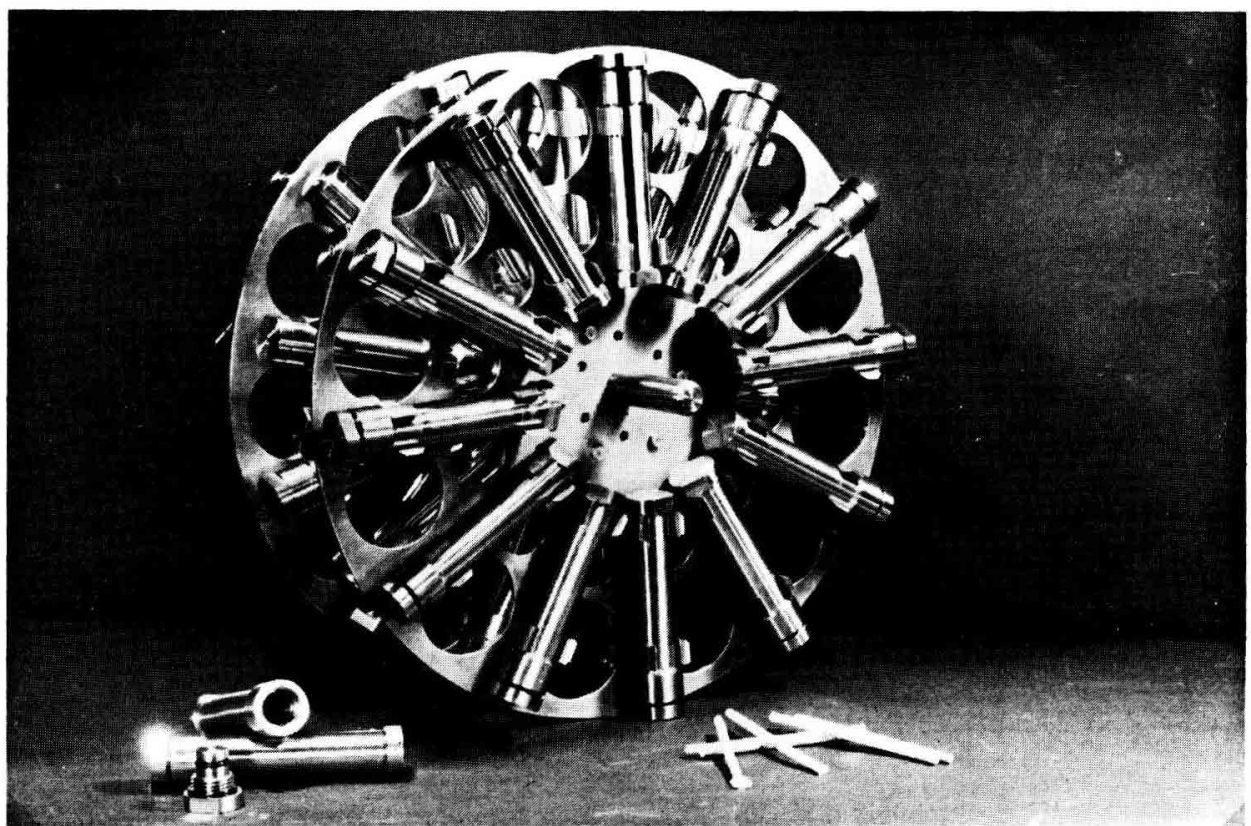

FIG. 2

(Cliché JANIN)

Support de 48 microlessiveurs

Two rotating wheels bearing 48 microdigesters

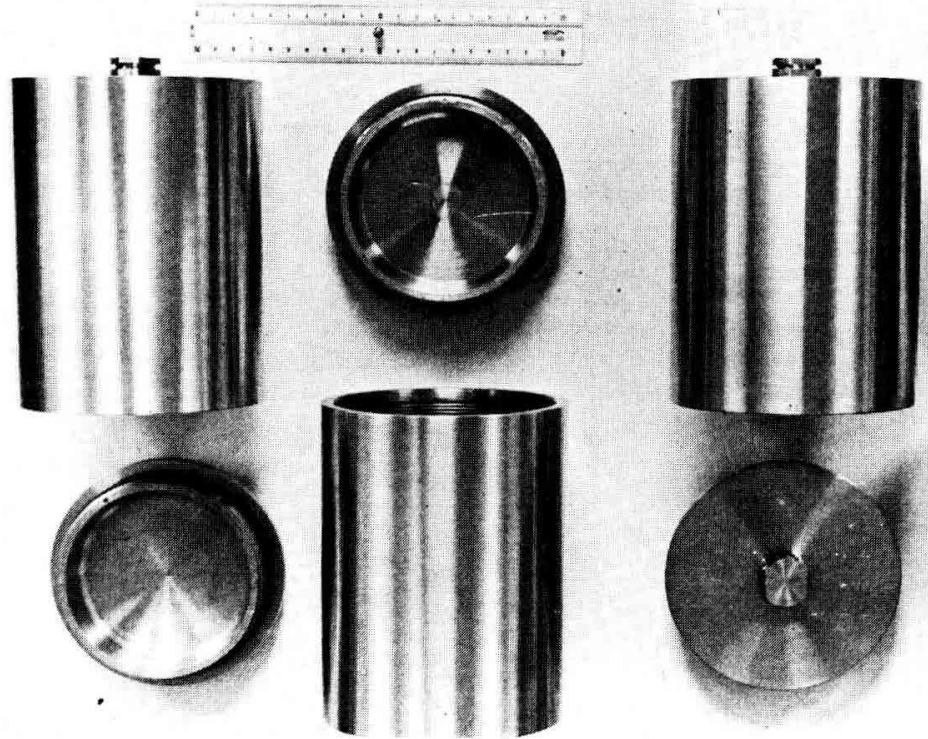

FIG. 3

4 gros lessiveurs de $1700 \mathrm{ml}$ de capacilé 4 macrodigesters $1700 \mathrm{ml}$ capacity

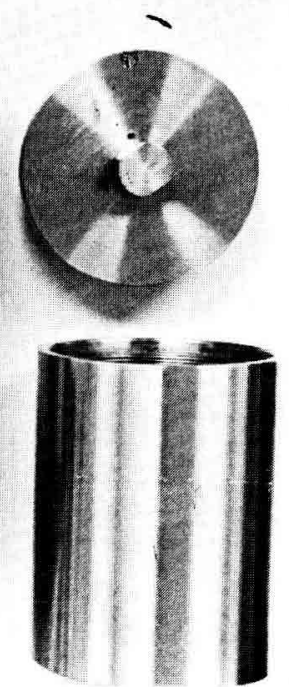

(Cliché Durand) 
Un circuit de refroidissement en tube de cuivre permet après les cuissons de ramener rapidement l'huile à la température du laboratoire pour lui éviter une détérioration trop rapide.

\subsection{2. - Macro et microlessiveurs}

Les macrolessiveurs d'un volume utile de $1700 \mathrm{ml}$ peuvent contenir $200 \mathrm{~g}$ de bois anhydre et sont comparables à ceux des laboratoires de contrôle industriels. Ils nous servent à établir la relation avec nos cuissons à échelle réduite (figure 2).

Les microlessiveurs d'un volume utile de $12 \mathrm{ml}$ permettent de traiter de $0,2 \mathrm{~g}$ à $2 \mathrm{~g}$ de bois. Dans le cas de très petits échantillons, une masselotte en acier inoxydable est introduite pour favoriser l'agitation plus vive des réactifs (figure 3).

\section{2. - Mode opératoire}

\subsection{1. - Préparation, conservation, dosage des liqueurs de cuisson}

La préparation de la liqueur se fait en une seule fois pour le volume nécessaire à une expérience comportant 20 ou 30 traitements journaliers de 48 microlessiveurs.

Les réactifs utilisés sont des produits purs de laboratoire : $\mathrm{NaOH}$ en pastilles et $\mathrm{Na}_{2} \mathrm{~S}, 9 \mathrm{H}_{2} \mathrm{O}$ cristallisé, et l'on procède par pesées des quantités nécessaires et ajustage des titres des solutions.

La conservation de la liqueur se fait à $-5{ }^{\circ} \mathrm{C}$ pour éviter l'oxydation des sulfures et la carbonatation de la soude dans le temps. La liqueur est répartie en autant de flacons de $500 \mathrm{~cm}^{3}$ que de cuissons journalières prévues. Chaque flacon est ramené à la température du laboratoire avant son emploi et son dosage. Le dosage journalier de la soude et du sulfure n'a jamais fait apparaître de différences significatives au cours de ces répétitions.

\subsection{2. - Distribution de la liqueur}

Une des constantes de la méthode de la cuisson Kraft est le rapport liqui$\mathrm{de} /$ bois (L/B) qui fixe la quantité de réactifs introduits et leur concentration au contact du bois. Dans la pratique courante $L / B$ est égal à 4 .

Chaque échantillon de bois étant différent, son poids anhydre est déterminé au dixième de milligramme et le volume de liqueur correspondant devrait être apprécié à $4 / 10^{\mathrm{e}}$ de $\mathrm{mm}^{3}$, ce qui est illusoire.

Nous avons donc choisi une burette automatique à piston de débit variable et dont la précision est de $2 \mathrm{~mm}^{3}$. Dans l'état actuel de la méthode cette précision est satisfaisante et, par ailleurs, elle permet d'éviter des variations largement plus importantes dues au manipulateur. 


\subsection{3. - Récupération des fibres par filtration}

Nous n'utilisons plus les creusets filtrants en verre fritté, d'où il était difficile de récupérer les fibres et qui nécessitaient deux pesées à l'état anhydre avant et après la filtration.

Nous avons retenu le système filtre Gelman à fermeture par anneau magnétique qui permet d'interposer une toile très fine de 300 Mesh Afnor sur laquelle toutes les fibres sont retenues; l'échantillon de fibres peut alors être aisément détaché de la surface, numéroté, porté à l'étuve à $102^{\circ} \mathrm{C} \pm 1^{\circ}$ et pesé à l'aide d'une balance dont la précision est le $1 / 10^{\mathrm{e}}$ de milligramme.

\section{3. - Contrôle de la qualité de la cuisson}

\section{1. - Micro indice de permanganate $\mathrm{KMnO}_{4} \mathrm{~N} / 10$}

La nécessité de disposer d'un micro indice de permanganate $\mathrm{KMnO}_{4} \mathrm{~N} / 10$ s'est fait sentir devant le poids toujours très réduit de fibres disponibles qui varie de $0,1 \mathrm{~g}$ à $0,75 \mathrm{~g}$.

Il existait certes un indice de Micro Kappa NF-T 12019 dérivé de l'Indice Kappa des pâtes NF-T 12018 et proposé pour la détermination des indices des pâtes en cours de blanchiment qui contiennent des taux de lignines résiduelles faibles et décroissants, mais son utilisation n'est possible que si l'on n'est pas limité du point de vue de la quantité de pâte.

Or nos échantillons de faibles poids contiennent des taux de lignines normaux des pâtes Kraft.

Il fallait donc adapter l'indice de $\mathrm{KMnO}_{4} \mathrm{~N} / 10 \mathrm{NF}-\mathrm{T} 12007$ à des poids de pâtes inférieurs au gramme en conservant les conditions opératoires prévues par la norme pour :

- la normalité du $\mathrm{KMnO}_{4} \mathrm{~N} / 300$ et de $\mathrm{H}_{2} \mathrm{SO}_{4} 4 \mathrm{~N} / 30$ au contact des fibres;

- le temps de réaction $5 \mathrm{~mm} \pm 10$ secondes.

Nous avons donc réduit le volume final du mélange de réaction et le nombre de $\mathrm{cm}^{3}$ de $\mathrm{KMnO}_{4} \mathrm{~N} / 10$ et $\mathrm{H}_{2} \mathrm{SO}_{4} 4 \mathrm{~N}$ initiaux dans la proportion du poids de pâte disponible. En effet, dans notre cas particulier, nous connaissons le poids exact de chaque échantillon après microcuisson : c'est le poids de fibres de chaque microlessiveur.

La linéarité de la consommation du $\mathrm{KMnO}_{4} \mathrm{~N} / 10$ dans les conditions de la norme NF-T 12007 en fonction du poids de fibres a été testé (figure 4) pour deux pâtes au sulfate dont les indices de permanganate $\mathrm{KMnO}_{4} \mathrm{~N} / 10$ étaient respectivement :

-- pin maritime (Pinus pinaster) : $27 \pm 0,5$;

- hêtre (Fagus silvatica) : $13,5 \pm 0,5$. 


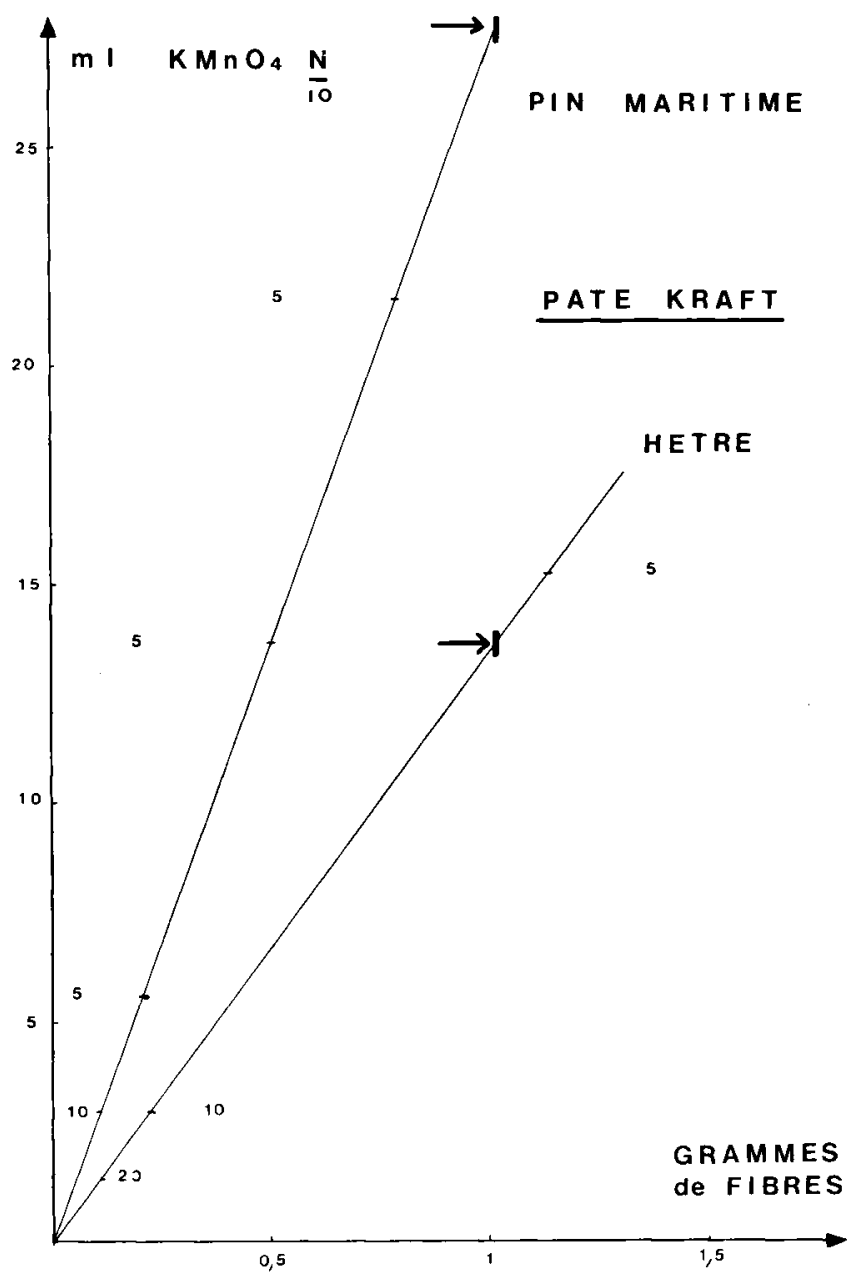

FIG. 4

Linéarité de consommation de $\mathrm{KMnO}_{4} \mathrm{~N} / 10$ en fonction du poids de pâte Kraft

Les flèches indiquent respectivement les indices de $\mathrm{KMnO}_{4} \mathrm{~N} / 10$ des pâtes pour 1 gramme de pâte :

Hêtre (fagus silvatica) : 13.5 .

Pin maritime (pinus pincaster) : 27,5.

Consumption linearity of $\mathrm{KMnO}_{4} \mathrm{~N} / 10$ versus the weight of Kraft pulp

The arrows show respectively the permanganate numbers values for 1 gram of pulp basis :

Beech (fagus silvatica) : 13,5.

Maritime Pine (pinus pinaster) : 27,5. 
Elle a été déterminée à l'aide d'échantillons de fibres de poids variable obtenus par dilution d'une suspension de fibres de 10 grammes par litre et par prélèvements successifs aux fins de dosage :

TABLEAU 1

Nombre d'échantillons dosés pour chaque poids de pâte

Samples number examined for each weight of pulp

\begin{tabular}{l|c|c|c|c}
\hline Poids de pâte & $0,1 \mathrm{~g}$ & $0,2 \mathrm{~g}$ & $0,5 \mathrm{~g}$ & $1 \mathrm{~g}$ \\
\hline Nombre d'échantillons & 20 & 10 & 5 & 5 \\
Pâte Kraft de Hêtre ............ & 10 & 5 & 5 & 5 \\
\hline
\end{tabular}

Les indices de permanganate $\mathrm{KMnO}_{4} \mathrm{~N} / 10$ des pâtes de microlessiveurs sont déterminés selon ces critères (G. JANIN, 1973). La correspondance entre l'indice de permanganate $\mathrm{KMnO}_{4} \mathrm{~N} / 10$ et l'indice Kappa des pâtes est par ailleurs établie (J.V. Hatton, 1975).

\section{2. - Dosage de liqueurs noires : consommation des réactifs}

Un volume de la liqueur noire de fín de cuisson est dilué dix fois à l'aide d'une solution alcoolique de $\mathrm{BaCl}_{2}\left(70 \mathrm{~g}\right.$ de $\mathrm{Bacl}_{2}$ dans $700 \mathrm{ml}$ d'eau $+300 \mathrm{ml}$ d'alcool éthylique à $96^{\circ}$. Ceci a pour effet de précipiter les acides organiques en sels de $\mathrm{Ba}$ et de donner par décantation ou filtration ou centrifugation une liqueur jaune clair où l'on peut doser la soude et le sulfure à l'aide d'un pH-mètre.

Pour estimer avec plus de précision la part de la $\mathrm{NaOH}$ libre et des sulfures présents par rapport à la quantité initiale, on peut ajouter du méthanal : $\mathrm{H}-\mathrm{CHO}$, qui se combine aux sulfures suivant les réactions globales :

$$
\begin{aligned}
2 \mathrm{HCHO}+\mathrm{S}^{--}+2 \mathrm{H}_{2} \mathrm{O} & \rightarrow\left(\mathrm{H}_{2}-\mathrm{C}-\mathrm{OH}\right)_{2} \mathrm{~S}+2(\mathrm{OH})^{-} \\
\mathrm{HCHO}+\mathrm{HS}^{-}+\mathrm{H}_{2} \mathrm{O} & \rightarrow \mathrm{H}_{2}-\mathrm{C}(\mathrm{SH}) \mathrm{OH}+(\mathrm{OH})^{-}
\end{aligned}
$$

Il se produit ainsi une libération stœchiométrique d’ions $(\mathrm{OH})^{-}$- correspondant aux ions $(\mathrm{HS})^{-}$et $\left(\mathrm{S}^{--}\right.$.

Ainsi, l'addition de méthanal à la fin du premier point équivalent (A) (figure 5) produit une augmentation du $\mathrm{pH}$ de 2,5 unités $\mathrm{pH}$ et rend ainsi plus nette l'observation du point (B) de la demi-équivalence des sulfures encore présents.

Ce dosage donne par différence la quantité d'alcali actif consommé au cours de la cuisson. 


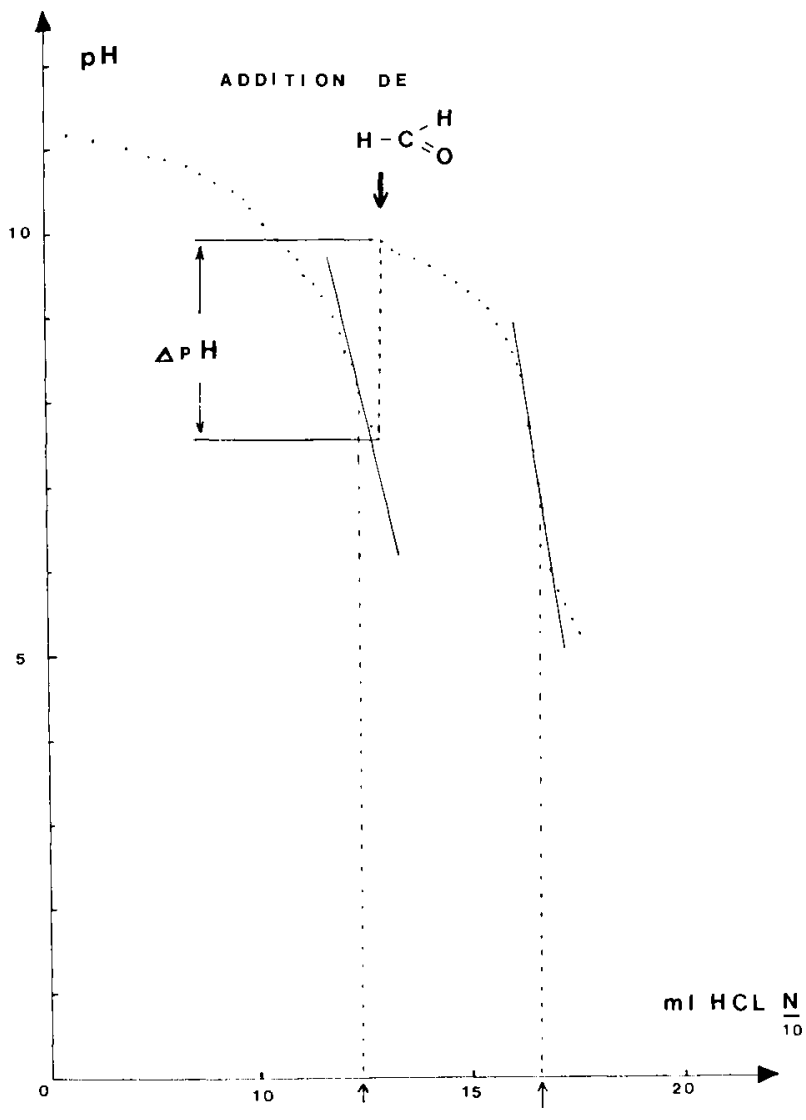

FIG. 5

Dosages séparés de $\mathrm{NaOH}$ et de $\mathrm{Na}_{2} \mathrm{~S}$.

$\triangle p H$ indique laugmentation du $\mathrm{pH}$ due à $\mathrm{Na} \mathrm{a}_{2}$ après addition de $\mathrm{H}$-CHO

$\mathrm{NaOH}$ and $\mathrm{Na} S$ separate titration.

$\triangle p H$ shows the $p H$ increase after the $H$-CHO addition

\section{3. - Les incuits}

On appelle «incuits» des parties de bois qui, après cuisson, ne sont pas. défibrées. Les incuits proviennent, soit d'un défaut de pénétration de la liqueur de cuisson dans le bois par suite de la présence d'un nœud, du voisinage d'un départ de branche, d'une accumulation ponctuelle de résines ou d'une surépaisseur locale dans un copeau de bois, soit d'un incident de manipulation : introduction d'un volume de liqueur insuffisant, température ou alcali actif trop faibles, défaut accidentel d'étanchéité au joint de fermeture du microlessiveur.

Lorsqu'ils se produisent, les incuits faussent l'expression du rendement et empêchent toute comparaison ultérieure. Ainsi, en plaçant les échantillons dans les mêmes conditions et en rejetant les carottes de sondage présentant des défauts apparents, la présence d'incuits devient tout à fait exceptionnelle ct fortuite. 
Dans le cas des microcuissons, les carottes de sondage présentent l'avantage unique, jamais réalisé dans d'autres traitements, de l'identité des dimensions des échantillons: ceci entraîne la régularité du mécanisme d’imprégnation des liqueurs et de la vitesse de dissolution des lignines et des hémicelluloses, et renforce la validité des comparaisons entre de nombreux échantillons.

\section{4. - Précision du rendement en fibres de la méthode de microcuisson}

Elle repose sur la détermination du poids auhydre du bois et des fibres. La précision relative de ce rendement $\Delta \mathrm{R}$ est donnée : en effet, si le rendement

$$
R=\frac{P \text { fibres }}{P \text { bois }} \times 100:\left(\frac{\Delta R}{R}\right)=\left(\frac{\Delta P \text { bois }}{P \text { bois }}\right)+\left(\frac{\Delta P \text { fibres }}{P \text { fibres }}\right)
$$

\section{1. - Pesée des échantillons}

La nécessité d'obtenir les poids anhydres de bois et de fibres pose le problème de la pesée en atmosphère ambiante du bois et des fibres dès la sortie de l'étuve en raison de leur affinité pour l'eau.

Nous avons choisi cette voie de manipulation parce qu'elle apporte une exécution plus rapide des nombreuses pesées successives à réaliser chaque jour de traitement : soit 48 pesées de bois, puis 48 pesées de fibres. Nous n'utilisons pas le passage dans un dessicateur pour le refroidissement des échantillons en préalable à la pesée parce que ces opérations alourdiraient très rapidement le mode opératoire.

L'étuve est placée au voisinage de la balance de précision (Sartorius 2002 MP1) pour diminuer au maximum le temps qui s'écoule entre le prélèvement de l'échantillon dans l'étuve, son positionnement sur le plateau de la balance et l'établissement de l'équilibre de la pesée et son affichage digital, temps qui demeure cependant de l'ordre de 15 secondes.

\section{2. - Reprise en eau des échantillons}

La mesure de la reprise en eau des échantillons de bois et de fibres a été faite en suivant l'augmentation de leur poids sur l'affichage électronique de la balance pendant 30 secondes comptées dès la sortie de l'étuve à $102^{\circ} \mathrm{C} \pm 1{ }^{\circ} \mathrm{C}$ (tableau 2).

Nous constatons que la reprise en eau se fait à des vitesses différentes pour le bois et pour les fibres compte tenu de la plus grande accessibilité de l'eau dans le cas des fibres. 
TABLEAU 2

Exemple de reprise en eau du bois et des fibres 15 s avant leur pesée

Water adsorption values of wood and fibers 15 s before weighted

\begin{tabular}{|c|c|c|c|}
\hline & \multicolumn{2}{|c|}{ Poids (en grammes) } & \multirow{2}{*}{$\begin{array}{l}\text { Adsorption d'eau } \\
10^{-1} \text { gramme/seconde/gramme } \\
\text { de bois }\end{array}$} \\
\hline & à $t_{1-n s}$ & à $t_{: 311 s}$ & \\
\hline Bois : carotte de sondage & 1,2235 & 1.2240 & 0,27 \\
\hline Fibres $\ldots \ldots \ldots \ldots \ldots \ldots$ & 0,6518 & 0,6529 & 1,12 \\
\hline
\end{tabular}

\section{3. - Estimation de la précision relative du rendement en pâte}

Nous devons nous placer dans l'hypothèse la plus défavorable pour tenir compte de la marge d'erreur la plus importante; ainsi, nous considérons que :

- le poids Po réel des échantillons à l'instant $t_{1}$ de la sortie de l'étuve est très difficile à déterminer d'une façon satisfaisante et, pour cela, nous supposerons que la reprise de poids entre l'instant $t_{10}$ et $t_{15}$ est linéaire et voisine de celle obtenue entre les instants $t_{15}$ et $t_{31 !}$. Nous majorons ainsi l'erreur systématique sur le poids des échantillons qui devient bien supérieure à la précision théorique de la balance;

- le calcul de la précision portera sur le poids maximum des échantillons couramment utilisé, soit $1,5 \mathrm{~g}$ de bois pour $0,75 \mathrm{~g}$ de fibres.

De ce fait, l'estimation de la reprise en eau des échantillons au cours de la pesée, tirée du tableau ci-dessus est de $6.10^{-1} \mathrm{~g}$ pour le bois et $12,6.10^{-4} \mathrm{~g}$ pour les fibres.

L'erreur relative sur le rendement devient alors :

$$
\left(\frac{\Delta \mathrm{R}}{\mathrm{R}}\right)=\frac{6 \cdot 10^{+}}{1,5}+\frac{12,6 \cdot 10^{-4}}{0,75} \# 2 \%
$$

Ainsi, pour un rendement de $50 \mathrm{p}$. 100, la précision obtenue est de donc de $\pm 0,1$ p. 100 dans le cas le plus défavorable ciû aux seules pesées du bois et des fibres en considérant que tous les autres facteurs de cuisson sont contrôlés, ce qui constitue un progrès appréciable. En effet, les influences déjà signalées de la préparation des échantillons (J.L. KeaYs et al., 1969, op. cit.), de leur séchage (A. Durand, 1975), de leur homogénéité et de leur nombre (J.L. KeAYS et al., 1969, op. cit.) sont écartées par l'emploi des carottes de sondage qui subissent des traitements uniformes avant leur cuisson.

\section{5. - Résultats}

Pour illustrer les applications de la méthode de microcuisson, nous examinerons sa reproductibilité au cours d'une même expérience, la relation existant entre elle et la méthode de cuisson à plus grande échelle, puis une étude relative aux facteurs 
physiques de la cuisson des bois et des paramètres concernant la localisation et la forme des échantillons dans l'arbre.

D'autres sources de variations influençant le rendement en pâte ayant pour origine le bois de compression (G. JANIN, 1972 a, op. cit.), l'orientement (G. JANIN, 1972 b ; A. Rahme, G. Janin, 1972) ont par ailleurs été décrites.

\section{1. - Reproductibilité des rendements}

Dans le cadre d'une étude de la «validité des tests précoces d'appréciation des caractères technologiques du bois de Douglas (Pseudotsuga menziesii) de M. THoBY (1975), l'échantillonnage était constitué de carottes de sondage prélevées dans 360 arbres âgés de 9 ans recouvrant 24 provenances de douglas Nord américains, à raison de 15 individus par provenance, et dans deux répétitions.

Pour tester les qualités papetières de ces différentes provenances à l'aide des microcuissons, Ies conditions suivantes ont été retenues :

— diagramme température-temps : 1 h 30 de $20^{\circ} \mathrm{C}$ à $170^{\circ} \mathrm{C}$

-. rapport liquide/bois

: 1 h 30 à $170^{\circ} \mathrm{C}$

- sulfidité

: 4

- alcali actif

: 25 p. 100

: 20 p. 100 en $\mathrm{Na}_{2} \mathrm{O}$

22 p. 100 en $\mathrm{Na}_{2} \mathrm{O}$

Deux alcalis actifs ont été choisis pour connaître les réponses à deux traitements d'intensité croissante en vue d'une sélectivité plus grande.

5.11. - Analyse de variance (SNEDECOR, 1934)

Les valeurs du test $\mathbf{F}$ de Snedecor tirées de l'analyse de variance et associées aux degrés de liberté sont les suivantes :

\section{Tableau 3}

Valeurs des tests $F$ entre provenances significatifs pour le rendement en pâte Valeurs des tests $F$ entre cuissons journalières non significatifs pour le rendement en pâte

Significant $F$ test values between provenances for the pulp yield

Unsignificant $F$ test values between daily cookings experiments for the pulp yield

\begin{tabular}{|c|c|c|}
\hline \multirow{2}{*}{ Facteur de variation } & \multicolumn{2}{|c|}{ Alcali actif } \\
\hline & $20 \%$ & $22 \%$ \\
\hline Provenances $: \mathbf{F}_{i=231}^{23} \ldots \ldots \ldots \ldots \ldots$ & $1,868\left(^{*}\right)$ & $1,923(* *)$ \\
\hline Répétitions journalières : $\mathrm{F}_{\text {: }}^{1: 4} \ldots$ & $1,417 \mathrm{NS}$ & $1,039 \mathrm{NS}$ \\
\hline $\begin{array}{l}(*) \text { Significatif à } 5 \% . \\
(* *) \text { Significatif à } 1 \% . \\
\text { NS : Non significatif. }\end{array}$ & & \\
\hline
\end{tabular}


Nous observons que les répétitions journalières n'entraînent pas de fluctuations statistiquement significatives pour le traitement des 24 provenances, ce qui nous assure une bonne reproductibilité (H.S. Gardner et D.W. EinspaHr, 1964).

\subsection{2. - Comparaison des deux traitements à 20 p. 100 et 22 p. 100 d'alcali actif}

Quelques éléments simples de statistique montrent les possibilités de comparaison qu'offre cette méthode en vue du choix de provenances pour leurs qualités papetières :

\section{Tableau 4}

Valeur moyenne, écart-type et coefficient de variation des rendements en pâte pour les deux alcali actifs

Mean, standard error and coefficient of variation of pulp yield for the both active-alkali

\begin{tabular}{cc|c|c|c}
\hline \multicolumn{2}{c|}{ Alcali actif } & Moyenne & Ecart-type & $\begin{array}{c}\text { Coefficient } \\
\text { de variation (\%) }\end{array}$ \\
\hline $20 \%$ & $\ldots \ldots \ldots \ldots \ldots$ & $\ldots \ldots$ & 1,01 \\
$22 \%$ & $\ldots \ldots \ldots \ldots \ldots$ & 47,0 & 1,085 & 2,14 \\
& 44,8 & $\ldots$ & $\ldots$ \\
\hline
\end{tabular}

Nous pouvons donc distinguer des provenances entre elles et choisir avec certitude la provenance ou le groupe de provenances qui donne des rendements en pâte supérieurs de 1 p. 100 à la moyenne des provenances, ce qui entraîne dès lors un gain appréciable.

\section{2. - Correspondance entre les rendements en pâte pour les macro et les microlessiveurs}

Un essai de comparaison de 28 individus provenant de clones, d'espèces pures ou d'hybrides différents du genre Populus et comprenant des hybrides Tremula $x$ Tremuloides $\mathrm{n}^{\prime \prime} 311$ et 312 nous a amené à utiliser des carottes de sondage et des copeaux de bois prélevés sur les mêmes tiges (LEMoINE, 1973).

Les conditions de cuisson étaient fixées à : 16 p. 100 d'alcali actif comptés en $\mathrm{Na}_{2} \mathrm{O}, 25$ p. 100 de sulfidité, un rapport liquide/bois de 4, et un diagramme températuretemps de : 1 h 30 de $20^{\circ} \mathrm{C}$ à $170^{\circ} \mathrm{C}$ et 1 h 30 à $170^{\circ} \mathrm{C}$.

La comparaison a été faite sur l'échantillonnage de bois suivant :

- macrolessiveurs : $200 \mathrm{~g}$ de bois provenant d'un disque de chaque arbre,

— microlessiveurs : 12 échantillons de $1 \mathrm{~g}$ à $1,5 \mathrm{~g}$ pour chaque arbre prélevés sur la même section transversale de $30^{\circ}$ en $30^{\circ}$.

Nous avons fait correspondre la moyenne du rendement des 12 microlessiveurs 
au rendement de chaque macrolessiveur pour le même arbre (figure 6); la droite de régression est :

$$
\mathrm{Y}_{\text {rendement en macro }}=0,911 \mathrm{X}_{\text {rendement moyen en micro }}+4,964
$$

avec un coefficient $r=0,832$ pour 28 couples significatif au seuil de 1 p. 1000 .

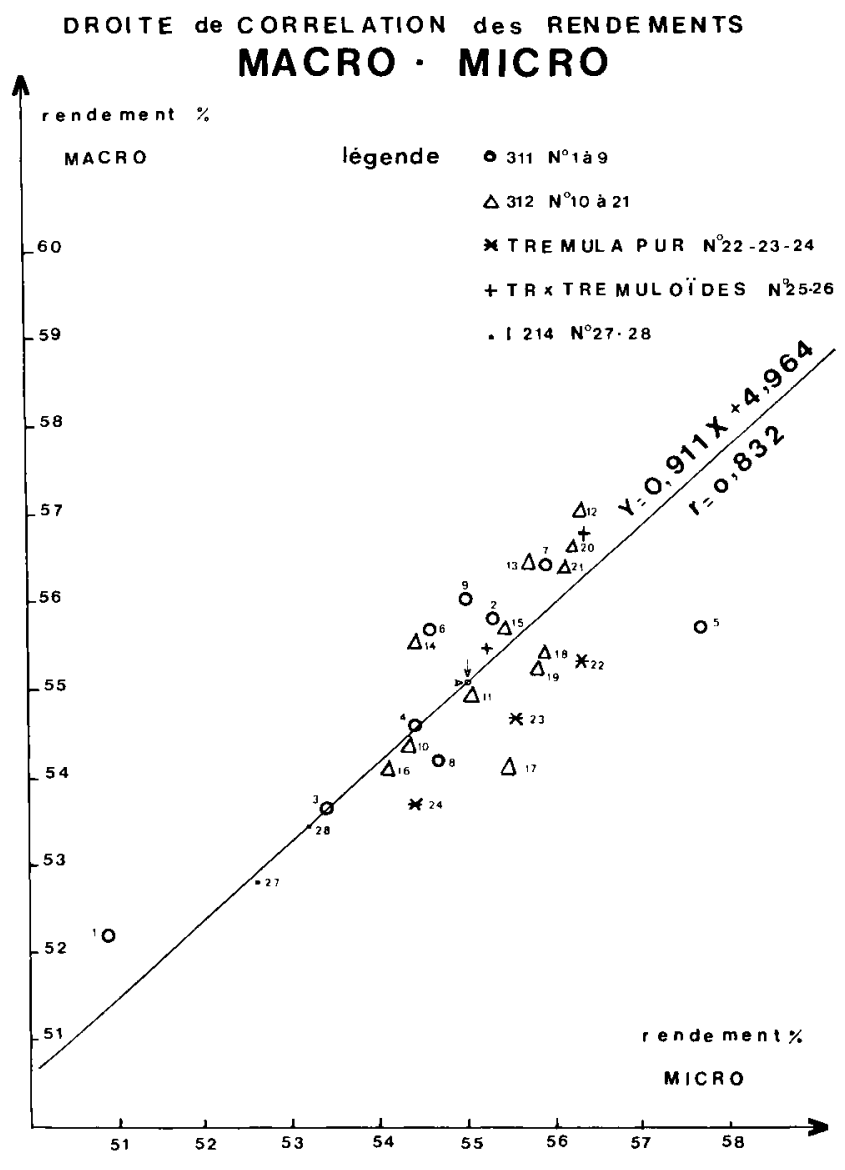

Fig. 6

Correspondance des rendements en pâte en macro et micro-lessiveurs

Macrodigesters and microdigesters relationships for pulp yield

5.3. - Influence des conditions de cuisson, de forme et de localisation des échantillons sur le rendement en pâte

Nous avons cherché à apprécier les effets sur le rendement en pâte des facteurs physiques de la cuisson papetière : température, échantillonnages de bois sous la 
forme de parallélépipèdes ou de carottes de sondage et à différentes positions dans la même section de l'arbre (figure 7).

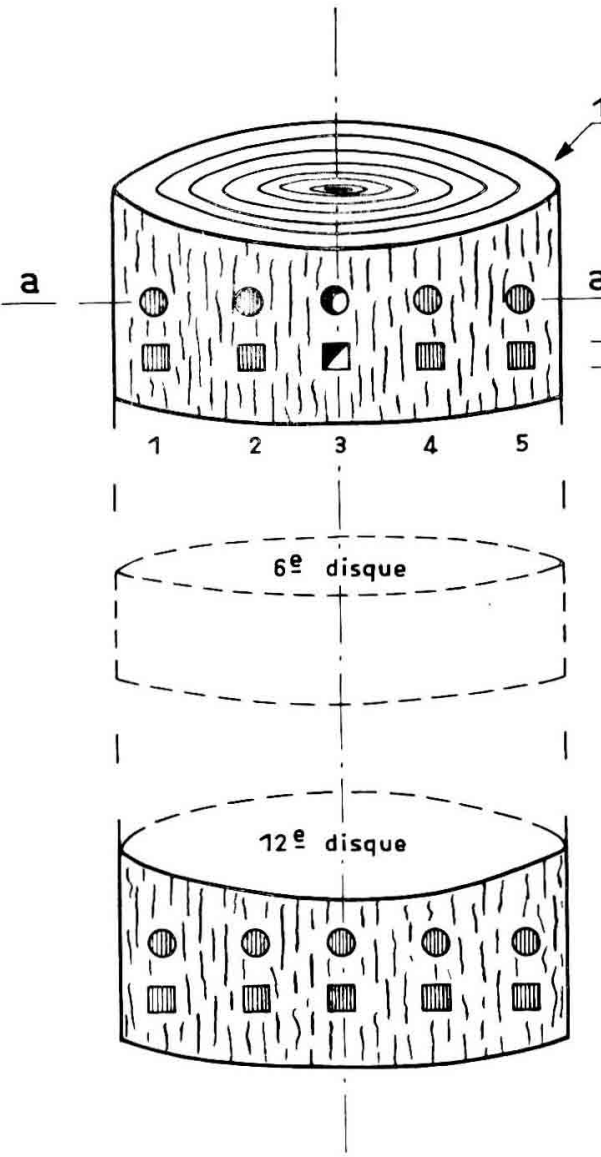

ELEVATION

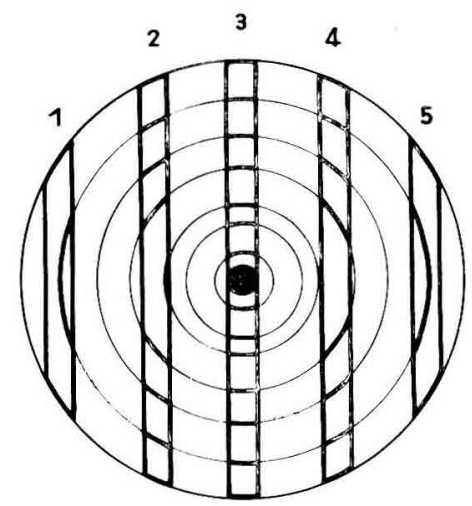

COUPE a

FIG. 7

Schéma du prélèvement de l'échantillonnage : carottes de sondage, parallélépipèdes, dans chaque disque d'un populus tremula

Sampling : increment cores, parallelepipeds in each disk of one Populus tremula

Ainsi, nous avons retenu :

- 3 températures de pallier : $160^{\circ}-167,5^{\circ}-170^{\circ}$,

- 2 formes d'échantillons : parallélépipédiques, carottes de sondage, 
- 5 positions :

1) externe,

2) intermédiaire,

3) diamétrale,

4) intermédiaire opposé,

5) externe opposé,

- 4 répétitions,

soit 120 échantillons.

Ces échantillons ont été prélevés dans un Populus tremula avec une tarière de Pressler d'une part, et d'autre part débités à la double scie avec un écartement de $5 \mathrm{~mm}$ après prélèvement des disques correspondants.

Le tableau de lanalyse de variance est le suivant :

\section{TABLEaU 5}

Analyse de variance portant sur 3 caractères de forme, température, position et leurs interactions

Variance analysis on three caracters : shape, temperature, position and their interactions

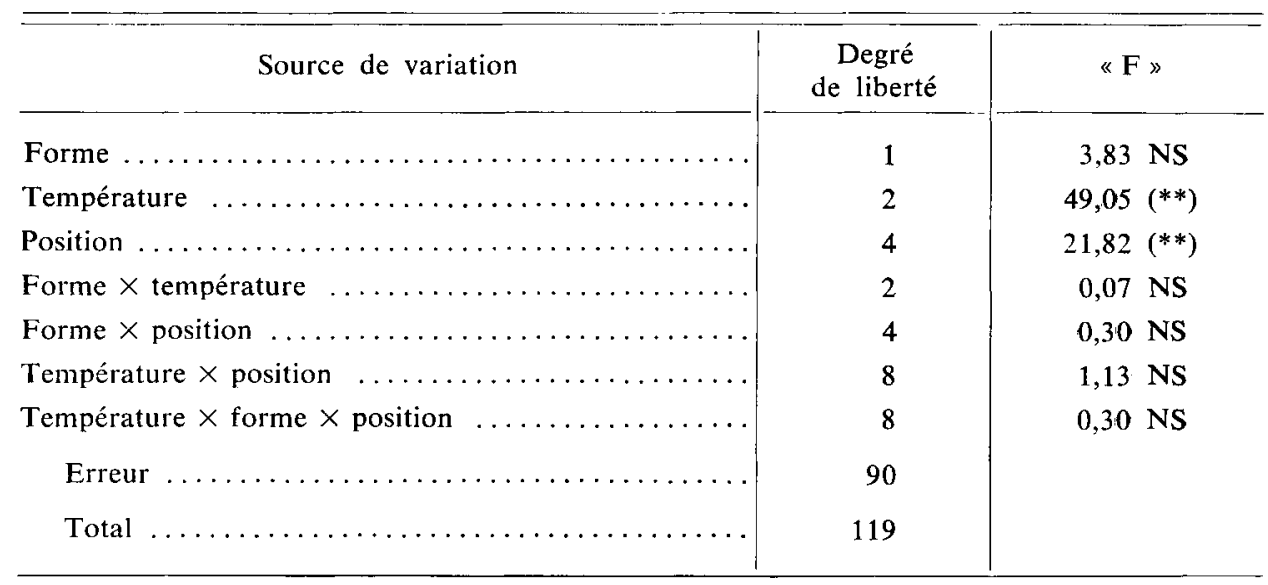

(**) Significaif à $1 \%$.

NS : Non significatif.

\section{Discussion}

La sensibilité de la méthode est mise en évidence par ces résultats.

La température, dont les effets sont connus par ailleurs, provoque une diminution du rendement lorsqu'elle s'élève pour une durée de pallier égale, et conduit ici à un abaissement significatif du rendement de $0,5 \mathrm{p} .100$ entre $167,5^{\circ}$ et $170^{\circ} \mathrm{C}$. La précision des résultats dépend étroitement de la régulation de la température. 


\section{TABleau 6}

Comparaisons de moyennes des rendements en fonction de la température Means pulp yicld comparison in terms of temperature

\begin{tabular}{ll|c|c|c}
\hline Température $\ldots \ldots \ldots \ldots$ & $160^{\circ}$ & $167,5^{\circ}$ & $170^{\circ}$ \\
\hline $\begin{array}{c}\text { Rendement moyen } \\
\text { échantillons }(\%)\end{array} \ldots \ldots \ldots$ & 55,2 & 53,3 & 52,8 \\
\hline
\end{tabular}

Comparaison :

$$
\begin{aligned}
& <\text { ppds }>0,50 \\
& <\text { ppds }>0,65
\end{aligned}
$$

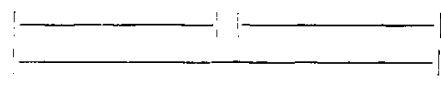

au seuil de $5 \%$ au scuil de $1 \%$

(ppds $=$ plus petite différence significative).

La position des échantillons sur la section transversale est importante; elle reflète à la fois la variation du rendement avec l'âge et la dissymétrie existant dans l'arbre :

TABleau 7

Comparaison des moyennes de rendements en fonction de la position

\begin{tabular}{|c|c|c|c|c|c|}
\hline Position & 1 & 2 & 3 & 4 & 5 \\
\hline $\begin{array}{c}\text { Rendement moyen } \\
\text { échantillons }(\%)\end{array}$ & 52,4 & 53,0 & 53,9 & 54,7 & 54,9 \\
\hline
\end{tabular}
Means pulp yield comparison in terms of position

Comparaison :

$$
\begin{aligned}
& <\text { ppds }>0,64 \\
& <\text { ppds }>0,84
\end{aligned}
$$

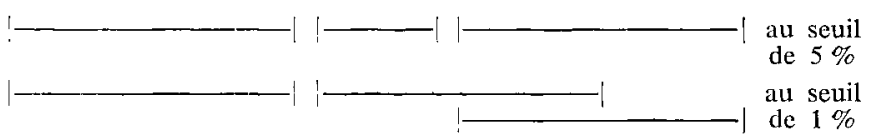

La moyenne générale $(53,8$ p. 100) coïncide avec la moyenne des échantillons suivant le plus grand diamètre. Cette remarque, jointe à notre expérience des microcuissons, nous amène à observer que :

- le prélèvement d'une carotte de sondage doit être diamétral,

- pour les comparaisons entre arbres de même situation, les carottes de sondage doivent avoir le même orientement.

La forme des échantillons n'influence pas le rendement si les dimensions axiales et tangentielles demeurent voisines : parallélépipèdes de $5 \mathrm{~mm}$ de côté et carottes cylindriques de $5 \mathrm{~mm}$ de diamètre. Nous pouvons ainsi travailler aussi bien avec de petits échantillons prélevés sur une section transversale après abattage d'un arbre qu'avec des carottes de sondage prises dans un arbre vivant. 
L'absence complète d'interaction significative du second ordre ct de celle de troisième ordre rend nos observations plus nettes : en effet, si la température et la position des échantillons sont définics pour une forme voisine, les rendements en pâte obtenus avec les microcuissons pourront être utilisés avec une certitude satisfaisante.

\section{6. - Conclusion : champs d'application de la méthode}

La facilité de mise en ceuvre de la méthode de microcuisson sur un très grand échantillonnage et de poids réduit permet d'évaluer statistiquement la valeur papetière des individus choisis, aussi bien dans des peuplements à l'état juvénile, dès l'âge de 5 ans, qu'à l'état adulte.

Nous pouvons ainsi estimer la variabilité individuelle et génétique des qualités papetières : rendement en fibres et, par extension, morphologie des fibres dans les tests de descendances et procéder au choix des familles donnant les meilleurs rendements comme à celui des provenances dans les tests de comparaison de provenances de sélection à clones (G. JANIN \& B. Letzelter, 1977), ou de classement d'espèces tropicales (G. Petroff \& M. Tissot, 1973).

Elle permet aussi l'étude de l'influence sur les mêmes qualités papetières de l'ensemble des facteurs de la production forestière : roche-mère, hétérogénéité du sol, climat, mode de sylviculture : espacement et taillis à courte rotation, fertilisation et âge des peuplements.

De plus, les aspects particuliers de lanatomie du bois : zones de bois de réactıon (de tension chez les feuillus et de compression chez les résineux), présence de nœuds, nature des écorces, jouent un rôle réel dans le rendement en pâte final.

Les variations de la structure interne des accroissements annuels pris individuellement ou par tranches successives déterminent le comportement du bois dans les traitements papetiers.

Les échantillons de bois de différentes espèces, prélevés sous forme de carottes de sondage à la tarière de Pressler, sont, dans un premier temps, étudiés pour la densité et les variations de la densité (H. Polge, 1978), la rétractibilité (A. Sesbou, G. Nepveu, 1978), la largeur des cernes, la teneur en matières extractibles, puis traités en microcuissons papetières et, ainsi, tous les critères de la qualité du bois et les caractères papetiers (M. El RHAZı \& G. JANIN, 1979) peuvent être mis en corrélation dans le but de définir le rôle joué par chacun d'eux dans la valeur papetière des espèces forestières.

Reçu pour publication en octobre 1979. 


\section{Summary \\ Micropulping technique improvement; pulp yicld precision}

The new micropulping equipment consists of an oil bath electrically heated equipped with to rotating wheels $(6$ r.p.m. $)$ wich bears 48 microdigesters $12 \mathrm{~cm}^{3}$ capacity in the same run; it makes up the major interest of this micropulping method. Thus a larger number of samples can be treated with very interesting combinations of factors : cooking times, alkali-active, liquor to wood ratio, tecause each microdigester is easily removable during the cooking process. Because the thin section, $5 \mathrm{~mm}$ diameter, and shape homogeneity of increment cores not any unscreened pulp occurs, or very seldom, and thus it allows accurate pulp yield comparison between samples. Moreover anatomic, genetic and forestry factors wich control the pulp yield and fiber length can be examined on particularly small samples and on those sampled from progeny test or provenances trials.

For the coocking comparison at the higher scale 4 macrodigesters 1.7 liter capacity are used too. The Kraft cooking liquors are prepared with laboratory pure products : $\mathrm{NaOH}$ pastilles and cristallized $\mathrm{Na}_{2} \mathrm{~S}, 9 \mathrm{H}_{3 n}$ soaked in pure water, cooled and stored at $-5^{\circ} \mathrm{C}$ throughout the cooking period for all the samples collected for the same experiment.

An adjusted volume of white liquor for each sample is added automatically at the nearest hundredth cubic centimeter. A suitable micropermanganate number $\left(\mathrm{KMnO}_{4} \mathrm{~N} / 10\right)$ is developped in connection with the same lignins content than the usually Kraft pulp and in proportion with the very samll weight of pulp pad analysed. The oven dry weight for both wood increment core and piece of pulp are determined within a 15 seconds interval after removed from the oven. Thus the weight is determinated without using a dessicator to conserve the versatility of the method when more than thousand samples must be carried out in the same experience.

The Kraft micropulping yield relative precision still reaches $\pm 0.1 \%$ in spite of the low water vapor sorption on the wood and pulp samples during this 15 seconds out of the oven assuming the worst. The cooking conditions and pulp yield reproducibility are quite good. Between 15 runs in two replications no effect was found for daily cooking experiments involving 720 samples $(r=0,832)$.

The pulp yield correlation from 28 trees of Populus species in the macro-digesters versus the mean of 12 micropulping yield from a whole disk of each tree is quite satisfactory $(r=0,832)$.

The elevation of the temperature : $160^{\circ} \mathrm{C}$. $167,5^{\circ} \mathrm{C}$. $170^{\circ} \mathrm{C}$. the well known cooking factor reduce the end pulp yield and the 2.5 " $\mathrm{C}$ last difference gives a significant $0.5 \%$ loss in pulp yield.

The different locations of samples accross the section shows the heterogeneity of pulp yield in a tree but the precisely diametrical sample is quite near the mean value for the entire disk.

For samples of similar sizes $(5 \mathrm{~mm}$ increment cores and $5 \mathrm{~mm}$ square section parallelepipeds) no significant effect on the pulp yield is observed.

The micropulping technique gives sound comparative results using $5 \mathrm{~mm}$ in diameter increment core sampling either mature trees or young trees even not elder than 5 years. The large number of samples and the wood quality data obtained yet on the increment cores samples can be correlated with the pulp wood qualities determined on the same samples.

\section{Références bibliographiques}

Blair R.L., Zobel B.J., Barker J.A., 1975. Predictions of gain in Pulp yield and tear strength in Young Loblolly pine through genetic increases in wood density. Tappi, 58 (1), 89-91.

Durand A. ,1975. Influence du séchage sur le rendement en pâte et diverses autres caractéristiques papetières. La Papeterie, $\mathrm{n}^{\circ} 2,78-81$. 
Einspahr D.W., van BuiJtenen J.P., Thode E.F., 1962. Wood and pulp properties as determined from Slash Pine increment core and whole tree measurement. Silvae genet., 11 (3), 68-77.

Einspahr D.W., van Buijtenen J.P., Peckham J.R., 1969. Pulping characteristics of ten-year Lobloliy pine selected for extreme wood specific gravity. Silvae Genet., 18 (3), 1969, 57-61.

El Rhaz. M., Janin G., 1979. Etude des effets de la sylviculture sur les caractéristiques papetières et physiques du bois d'Eucalyptus camaldulensis traité en taillis à moyenne rotation de 12 ans. A paraître dans A mln. Rech. forest. Maroc.

Gardner H.S., EINSPAhr D.W., 1974. Reproductibility of micropulping wood samples. Tappi, 47 (7).

HATTON J.V., 1975. Kappa number - permanganate number relationship for softwood and hardwoods. Tappi, 58 (10), 150-151.

JANING G., 1972 :

a) Microcuissons papetières. Méthode adaptée aux recherches forestières portant sur la détermination des caractéristiques papetières individuelles sur arbres vivants à l'aide d'échantillons de bois dont le mode de prélèvement, l'aspect et le poids ne sont pas usuels. La Papeterie, n" 3 , mars.

b) I.e diagramme polaire différentiel des rendements, nouveau critère d'homogénéité des bois all point de vue des propriétés papetières. Document à distribution limitée, Station de Recherches sur la Qualité des Bois, C.N.R.F., n" 1972/8.

JANIN G., 1973. Détermination des diagrammes polaires des rendements et des indices de permanganate sur carottes droites et obliques. Essais de microclassage sur carottes obliques. Document à distribution limitée, Station de Recherches sur la Qualité des Bois, C.N.R.F., n" 1973/4.

Janin G., Letzelter B., 1977. Cannes de Provence : détermination de l'échantillon moyen d'une tige en vue d'une sélection clonale. La Papeterie, n" 9, septembre.

Keays J.I.,. Hatton J.V., CORTEZ R.R., 1969. The precision of laboratory Kraft pulp yield determination. Tappi, 52 (5), 904-912.

Lemoine M., 1973. Amélioration des peupliers de la section Leuce sur sols hydromorphes. Thèse Docteur-Ingénieur. Université Nancy $I$.

Manville J.F., HUNT K., 1977. Precision Kraft pulping of small Douglas fir wood samples. Tappi. 60 (4), 138-139.

PetrofF G., Tissot M., 1973. Traitement par microtests de huit bois feuillus tropicaux et comparaison avec un traitement classique. Rapport du C.T.F.T., Division de Cellulose et Papier, mars.

Polge H., 1978. Fifteen years of wood radiation densitometry. Wood Sci. Techn., 12, 187-196. $\log y, 12,187-196$.

Rahme A., Janin G., 1972. Etude de la variabilité des rendements en fibres sur Pinus brutia. Document à distribution limitée, Station de Recherches sur la Qualité des Bois, C.N.R.F., n" 1972/3.

Тнову M. Validité de tests précoces d'appréciation des caractères technologiques du bois de Douglas. Application à une plantation comparative de 24 provenances de Douglas (Peyrat-le-Château) en liaison avec des caractères morphologiques et auxométriques. Rapport de stage E.N.I.T.E.F., Station de Recherches sur la Qualité des Bois, C.N.R.F., juin 1975.

Thode E.F., Peckham J.R., Daleski E.J., 1961. An evaluation of ceriain laboratory pulping methods. Tappi, 44 (2), 81-88.

Sesbou A., NePVeu G., 1978. Variabilité infraspécifique du retrait avec collapse et de la densité du bois chez Eucalyptus camaldulensis. Amn. Sci. For'st., 35 (3), 237-263.

SNEdeCOR G.W., 1934. Calculation and interpretation of analyses of variance and covariance. A.M.E.S., I.O.W.A. 